\title{
Turbo detection based on signal simplicity and compressed sensing for massive MIMO transmission
}

\author{
Zahran Hajji Karine Amis Abdeldjalil Aïssa-El-Bey \\ IMT Atlantique, UMR CNRS 6285 Lab-STICC, UBL, F-29238 Brest, France \\ Email: Firstname.Lastname@imt-atlantique.fr
}

\begin{abstract}
In this paper, we address the problem of large MIMO detection assuming QAM constellations. We show that the QAM signal becomes a simple signal (that is to say a bounded signal with extreme elements equal to the inferior and superior bounds [1]) after a real transformation. Based on this property, we present a low complexity detection algorithm which significantly outperforms classic algorithms such as zero forcing (ZF) and minimum mean square error (MMSE) algorithms. The proposed detection technique is based on a quadratic programming criterion whose constraints ensure that the detected vector is simple. We implement it successfully in an underdetermined MIMO system (the number of observations is less than the number of sources) and we show the necessary conditions of success detection. Then we consider an outer forward error correcting (FEC) code and we propose a turbo detection scheme. Based on the investigation of the output detector statistics in [2], we propose a symbol to binary converter (SBC) which can feed the FEC decoder with reliable output. On the other side, from the second iteration, the detection scheme resorts to a regularized quadratic criterion so that the searched vector draws near to the estimate resulting from the FEC decoder output. Simulation results show the efficiency of the proposed scheme.
\end{abstract}

Index Terms-Source separation, massive MIMO, turbodetection

\section{INTRODUCTION}

In previous generations of wireless communications, orthogonal multiple access schemes were used in order to serve the cellular users and allocate the radio resources among them. While $1 \mathrm{G}$ used frequency division multiple access (FDMA), $2 \mathrm{G}$ is based mostly on time division multiple access (TDMA) and $3 \mathrm{G}$ exploits code division multiple access (CDMA). In order to increase the spectrum efficiency, orthogonal frequency division multiple access (OFDMA) was adopted in the 4G. In these schemes, orthogonal radio resources are allocated to avoid the inter-user interference and provide an acceptable multiplexing gain with reasonable complexity.

However, the predicted exponential growth in the number of mobile connected machines and the traffic of data they represent motivate $5 \mathrm{G}$ designers to look for new technologies and approaches to address the mounting demand. It was theoretically demonstrated that the previous schemes cannot achieve the sum-rate capacity of multiuser wireless systems and the maximum number of supported users is limited by the total amount of orthogonal resources [3]. To overcome

The authors would like to thank the Brittany Region and PRACOM for their financial support. this problem and in order to support massive connectivity of users and devices, enhanced technologies are needed.

The massive MIMO is considered as a potential candidate to address the challenges of $5 \mathrm{G}$. The idea is to implement a large number of antennas to better exploit the spatial diversity so as to provide higher throughput under spectrum limitations. In this paper, we consider the detection problem in massive MIMO technology. Rather than dealing with interuser interference by designing proper transmitting schemes as Orthogonal Multiple Access (OMA), the proposed algorithm jointly decodes the useful signal and the interfering signal before selecting the source of interest.

Usual detection algorithms cannot be applied to massive MIMO systems. The optimum maximum likelihood (ML) detection fulfills the diversity requirement but its complexity is obviously too huge. The suboptimal ML-like sphere decoding (SD) technique [4] involves an exhaustive search within the hypersphere whose dimensions remain high in the large-scale MIMO case, yielding computationally-unsolvable detection. Linear solutions such as minimum mean square error (MMSE) [5] and zero-forcing (ZF) have low computation complexity at the expense of a high performance-loss. In previous works, the compressed sensing (CS) techniques have attracted a considerable attention. They suggest that it may be possible to surpass the traditional limits of the sampling theory. Thanks to a sparse transformation of the received signal and using the source separation techniques such as the basis pursuit (BP) which looks for the sparsest solution vector, it becomes possible to recover successfully the desired signal [6], [7], [8].

An outer FEC is usually applied before the modulation. Turbo-like receivers based on iterative information exchanges between its components (detection, decoder, channel estimation, synchronisation, ...) were proved efficient to achieve near-optimal performance. To that purpose, the authors proposed in [9] to associate a CS-based detection with a softdecision decoder within an iterative process based on a regularized detection criterion combined with a judicious sparse formulation of the detector output.

In this paper, we exploit the QAM signal simplicity (all its elements are bounded) [10] and we resort to CS techniques to propose a low-complexity detector adapted to underdetermined large MIMO systems. Contrary to [6], [7], [8], except the realvalued formulation of the problem, no signal transformation is required. We first present a quadratic criterion with bounded 
constraints to recover the received signal. Then, from the analytical detector output statistics, we define a reliable soft input of the FEC decoder. Finally we propose an iterative receiver with a regularized detection optimization criterion.

This paper is organized as follows. Section II describes the MIMO transmission model and its real-valued formulation. Section III deals with the detection problem in the MIMO case. We detail the detector optimization criterion and its analytical output probability density function. Section IV describes how the detector output statistics is exploited to exchange soft information with a FEC decoder. In Section V, we compare the proposed detector to MMSE-based equalizers both in the uncoded case and in the coded case. Finally, Section VI concludes the paper.

Notations: boldface upper case letters and boldface lower case letters denote matrices and vectors, respectively. For the transpose, transpose conjugate and conjugate matrices we use $(\cdot)^{T},(\cdot)^{H}$ and $(\cdot)^{*}$, respectively. $\otimes$ is the Kronecker product. $\boldsymbol{I}_{p}$ is the $p \times p$ identity matrix and $\mathbf{1}_{p}$ is the all-one size- $p$ vector.

\section{SYSTEM MODEL}

Let us consider a $K$-user MIMO transmission over a flat fading channel, where each user has $N_{t}$ transmit antennas and let $N=K \times N_{t}$. At the base station side, we assume $n$ equivalent receive antennas (base station cooperation through the base station controller is possible) with $n \leq N$. We assume a perfect knowledge of the channel state information (CSI) at the base station. In this paper, we focus on the uplink of the communication system. Under the above assumptions, the received signal can be modelled as:

$$
\boldsymbol{y}=\boldsymbol{H} \boldsymbol{x}+\boldsymbol{z},
$$

where $\boldsymbol{H}$ is an $n \times N$ complex random channel matrix, $\boldsymbol{x}$ is the $N \times 1$ complex data vector, and $\boldsymbol{z}$ is the $n \times 1$ complex circularly symmetric additive Gaussian noise vector with zero mean and a covariance matrix equal to $\sigma^{2} \boldsymbol{I}$. We assume that the components of $\boldsymbol{x}$ belong to an $M$-QAM modulation alphabet such that $M=p^{2}$ (square QAM constellation). The modulation alphabet is denoted by $\mathcal{Q}=\left\{q_{1}, q_{2}, \ldots, q_{M}\right\}$. We associate to $\mathcal{Q}$ the symbol vector $\boldsymbol{q}=\left[q_{1}, q_{2}, \ldots, q_{M}\right]$ where $q_{i}=a_{i}+j b_{i}, i \in\{1, \ldots, M\}$ with $\left(a_{i}, b_{i}\right) \in \mathcal{A} \times \mathcal{A}$ and $\mathcal{A}=\left\{\alpha_{1}, \alpha_{2}, \ldots, \alpha_{p}\right\}$.

We transform first the complex-valued system into an equivalent real-valued system, which reads

$$
\underline{y}=\underline{H x}+\underline{z},
$$

\footnotetext{
where $\underline{\boldsymbol{y}}=\left(\begin{array}{c}\Re(\boldsymbol{y}) \\ \Im(\boldsymbol{y})\end{array}\right), \underline{\boldsymbol{H}}=\left(\begin{array}{cc}\Re(\boldsymbol{H}) & -\Im(\boldsymbol{H}) \\ \Im(\boldsymbol{H}) & \Re(\boldsymbol{H})\end{array}\right), \underline{\boldsymbol{x}}=$ $\left(\begin{array}{c}\Re(\boldsymbol{x}) \\ \Im(\boldsymbol{x})\end{array}\right)$ and $\underline{\boldsymbol{z}}=\left(\begin{array}{c}\Re(\boldsymbol{z}) \\ \Im(\boldsymbol{z})\end{array}\right)$.

In this real-valued system, the first $N$ components of $\underline{x}$ are the real parts of the symbols and the imaginary parts are mapped to the next $N$ components.
}

\section{Detection of the Mimo signal via Euclidean DISTANCE MINIMIZATION}

\section{A. Proposed method definition and theoretical analysis}

In this section, we describe our technique to recover the real valued-vector $\underline{x}$. Considering the proposed real-valued formulation, the $[\mathrm{ML}]$ problem reads:

$$
\left(P_{M L, 2}\right): \underset{x}{\arg \min }\|\underline{\boldsymbol{y}}-\underline{\boldsymbol{H} \boldsymbol{x}}\|_{2} \quad \text { subject to } \quad \underline{\boldsymbol{x}} \in \mathcal{A}^{2 N} .
$$

This problem is NP-hard with high order of complexity. To circumvent this problem, we transform the optimization problem to a convex one by relaxing the constraints. In [8], the authors proposed to decompose the vector $\underline{x}$ as $\underline{x}=\boldsymbol{B}_{\mathcal{A}} s$ with $\boldsymbol{B}_{\mathcal{A}}=\boldsymbol{I}_{2 N} \otimes\left[\alpha_{1}, \alpha_{2}, \ldots, \alpha_{p}\right]^{T}$ and they defined a quadratic optimization criterion to look for the sparsest vector $s$ that minimizes the euclidean distance. Unfortunately, the complexity of the resulting algorithm increases with the constellation size, which becomes problematic for high-order modulations. In this paper, we exploit the simplicity property of QAM modulations to define an optimization problem whose complexity is independent of the constellation size and lower compared with [8]. A vector is considered to be simple when it has some elements equal to the extremes of the constellation. Taking advantage of the simplicity of the vector $\underline{\boldsymbol{x}}$, its components belong to the interval $\left[\alpha_{1}, \alpha_{p}\right]$ and then it can be decomposed as $\underline{\boldsymbol{x}}=\boldsymbol{B}_{\alpha} \boldsymbol{r}$ where $\boldsymbol{B}_{\alpha}=\boldsymbol{I}_{2 N} \otimes\left[\alpha_{1}, \alpha_{p}\right]^{T}$ and $r \in[0,1]^{4 N}$. We take into account just the extremes because all the elements can be written as a combination of them. Based on this decomposition, we propose to solve the following optimization problem:

$$
\begin{array}{r}
\left(P_{S I, 2}\right): \underset{r}{\arg \min }\left\|\underline{\boldsymbol{y}}-\underline{\boldsymbol{H}} \boldsymbol{B}_{\alpha} \boldsymbol{r}\right\|_{2} \quad \text { subject to } \\
\boldsymbol{B}_{1} \boldsymbol{r}=\mathbf{1}_{2 N}, \\
\boldsymbol{r} \geq 0,
\end{array}
$$

where $\boldsymbol{B}_{1}=\boldsymbol{I}_{2 N} \otimes \mathbf{1}_{2}$. The new optimization problem $\left(P_{S I, 2}\right)$ is a quadratic programming model. The linear equality constraint combined with the positivity constraint impose that the detected vector will be minored by $\alpha_{1}$ and majored by $\alpha_{p}$. The criterion $\left(P_{S I, 2}\right)$ can be optimized by the simplex [11] or the interior point methods[12]. In this paper, we consider the algorithms based on the interior point methods. These algorithms start by finding an interior point of the polytope defined by the constraints and then proceed to the optimal solution by moving inside the polytope.

In order to fix the acceptable range of values of the MIMO system dimensions, we consider the noiseless case and search for the conditions of solution uniqueness from the success probability. To that purpose, in Fig.1 we plotted the phase diagrams which represent the successful detection probability as a function of the ratio of the system dimensions $\frac{n}{N}$. It can be observed that the success probability is higher than $50 \%$ when $\frac{n}{N} \geq \frac{p-1}{p}$ and tends to 1 when $\frac{n}{N}$ increases with a higher convergence rate for large values of $N$ due to a better exploitation of the system diversity. We deduce that 


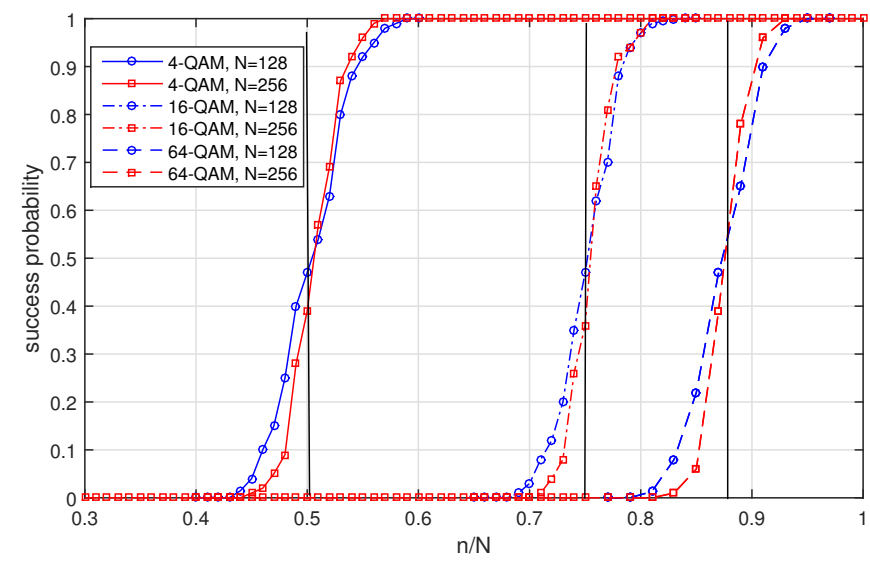

Fig. 1: Phase diagrams of the proposed detector for $M$-QAM with $M=4,16,64$ and $N=128,256$.

the proposed detector can be implemented in underdetermined systems which satisfy the following condition: $\frac{n}{N}>\frac{p-1}{p}$ [2].

In practice, an outer FEC will be used before the modulation and the detector will be interfaced with a soft-decision decoder. It is necessary to deliver reliable information to the FEC decoder. To that purpose we study the statistics of the detector output. As calculated in [2] and denoting $\hat{\boldsymbol{r}}$ the result of the optimization problem, the components of $\underline{\hat{\boldsymbol{x}}}=\boldsymbol{B}_{\alpha} \hat{\boldsymbol{r}}$ follow a censored normal distribution which can be seen as a combination of binary distributions on the bounds and Gaussian ones in the interior. The probability density function of the detector output components is given by [2]:

$$
f_{\underline{\hat{\boldsymbol{x}}}_{k}}(x)=\frac{1}{p} \sum_{\ell=1}^{p} f_{\underline{\hat{\boldsymbol{x}}}_{k} \mid \boldsymbol{x}_{k}=\alpha_{\ell}}(x),
$$

with

$$
\begin{aligned}
& f_{\underline{\hat{x}}_{k} \mid \boldsymbol{x}_{k}=\alpha_{\ell}}(x)=\left(\frac{1}{2} \operatorname{erfc}\left(\frac{\alpha_{\ell}-\alpha_{1}}{\sqrt{2} \sigma_{\underline{\underline{x}}}}\right) \delta_{\alpha_{1}}(x)\right. \\
& +\frac{1}{2} \operatorname{erfc}\left(\frac{\alpha_{p}-\alpha_{\ell}}{\sqrt{2} \sigma_{\underline{\hat{x}}}}\right) \delta_{\alpha_{p}}(x) \\
& \left.+\frac{1}{\sqrt{2 \pi} \sigma_{\underline{\hat{x}}}} \exp \left(-\frac{\left(x-\alpha_{\ell}\right)^{2}}{2 \sigma_{\underline{\hat{x}}}^{2}}\right) \mathbb{1}_{\left[\alpha_{1}, \alpha_{p}\right]}(x)\right) .
\end{aligned}
$$

and

$$
\sigma_{\underline{\hat{x}}}^{2}=\sum_{k=0}^{2 n-2}\left(\begin{array}{c}
2 N \\
k
\end{array}\right)\left(\frac{1}{p}\right)^{2 N-k}\left(\frac{p-1}{p}\right)^{k} \frac{2 n \sigma^{2}}{2 n-k-1},
$$

where $\delta_{\alpha}(x)$ is the Dirac delta function concentrated at $\alpha$ and $\mathbb{1}_{\Omega}(x)$ is the indicator function of the subset $\Omega$.

Then, we check that the simulated histogram of the detection output is in accordance with the theoretical statistical distribution. In Figs. 2 and 3, we observe that the theoretical distribution coincides with the simulated histogram for the different system dimensions and different SNR values, which supports the validity of the analysis.

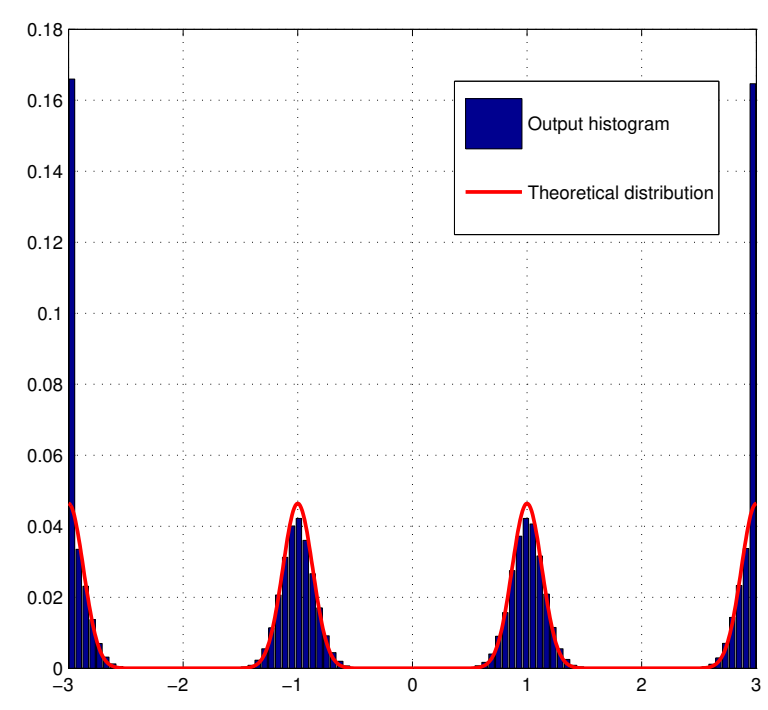

Fig. 2: Output statistics for $64 \times 64$ systems with 16-QAM and $S N R=30 d B$.

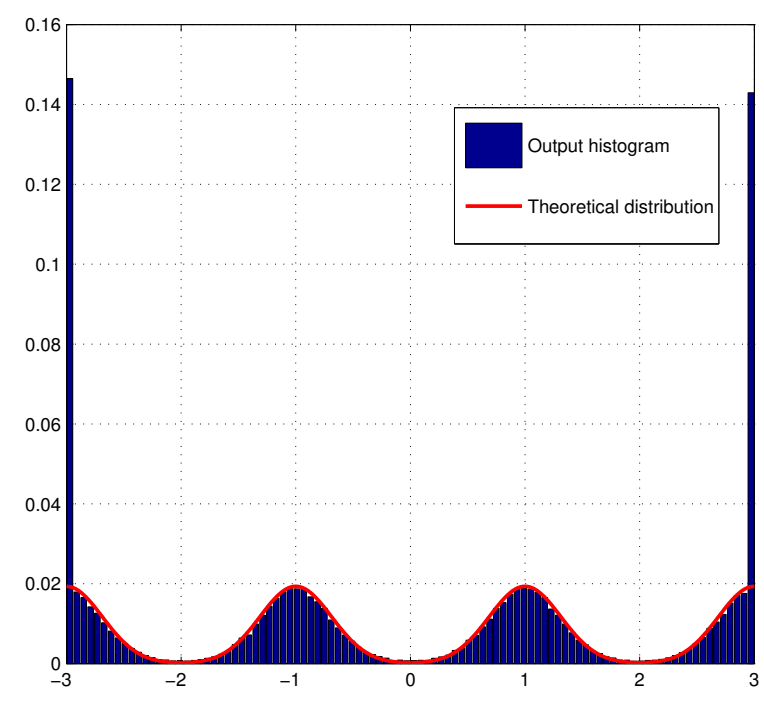

Fig. 3: Output statistics for $16 \times 16$ systems with 4-QAM and $S N R=20 d B$.

\section{B. Complexity analysis}

In Table I, we summarize the complexity order of the proposed detector $\left(P_{S I, 2}\right)$, the MMSE, the MMSE-Successive Interference Cancellation (MMSE-SIC) [13] and the SD nearoptimal detector. The SD detector is a high-complexity detector especially when the modulation order or the number of antennas increase, it is the least cost efficient. The MMSEbased detector consists of one matrix inversion and some matrix multiplications and additions. The MMSE-SIC adds some order of complexity. According to the complexity analysis 
TABLE I: Computational cost with the interior point method.

\begin{tabular}{|c|c|c|c|}
\hline & Iteration number & Computational cost per iteration & Total \\
\hline MMSE & 1 & $O\left(N^{3}\right)$ & $O\left(N^{3}\right)$ \\
\hline MMSE-SIC & 1 & $O\left(N^{3}\right)+O\left(M N^{2}\right)+O\left(M^{2} N\right)$ & $O\left(N^{3}\right)+O\left(M N^{2}\right)+O\left(M^{2} N\right)$ \\
\hline$\left(P_{S I, 2}\right)$ & $O(\sqrt{N})$ & $O\left(N^{2.5}\right)$ & $O\left(N^{3}\right)$ \\
\hline SD & 1 & $O\left(\sqrt{M^{N}}\right)$ & $O\left(\sqrt{M^{N}}\right)$ \\
\hline
\end{tabular}

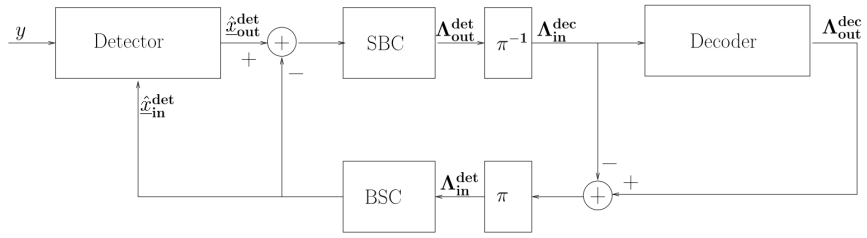

Fig. 4: Turbo detection scheme

in the case of determined MIMO systems, $\left(P_{S I, 2}\right)$ achieves the same order of complexity compared to the MMSE-based methods.

\section{Proposed turbo Detection SCHEME}

\section{A. Iterative receiver and soft-output detection}

In this section, our objective is to associate the proposed soft-output detector with a FEC decoder and make them work in an iterative manner (see Fig. 4). We consider that the binary stream is FEC encoded, then randomly interleaved before being converted into QAM symbols and passed through a serial-to-parallel converter.

Let $m=\log _{2}(\sqrt{M})$ and $c$ be the length- $m N$ coded and interleaved binary information sequence at one channel use. Let also $\psi$ be the binary-to-symbol conversion defined as:

$$
\psi:\left[\begin{array}{llll}
c_{k m} & c_{k m+1} & \ldots & c_{(k+1) m-1}
\end{array}\right] \in\{0,1\}^{m} \mapsto \underline{x}_{k} \in \mathcal{A}
$$

and $\boldsymbol{c}^{(j)}=\psi^{-1}\left(\alpha_{j}\right)$.

Using $\underline{\hat{x}}_{\text {out }}^{\text {det }}$, the symbol to binary converter (SBC) computes the $\log$ likelihood ratio on the $i$-th bit associated to the $k$-th symbol, denoted by $\Lambda_{\text {out }}^{\text {det }}$ and defined as:

$$
\begin{array}{r}
\Lambda_{\text {out }}^{\text {det }}(k m+i)=\log \left(\frac{\operatorname{Pr}\left(b_{k m+i}=1 \mid y\right)}{\operatorname{Pr}\left(b_{k m+i}=0 \mid y\right)}\right) \\
=\log \left(\frac{\left.\sum_{\alpha_{j} \in \mathcal{A}_{i, 1}} f_{\underline{\hat{x}}_{k} \mid \underline{x}_{k}=\alpha_{j}}\left(\underline{\hat{x}}_{\text {out }, k}^{\text {det }}\right) \operatorname{Pr}\left(\underline{x}_{k}=\alpha_{j} \mid \Lambda_{i n}^{\text {det }}\right)\right)}{\left.\sum_{\alpha_{j} \in \mathcal{A}_{i, 0}} f_{\underline{\hat{x}}_{k} \mid \underline{x}_{k}=\alpha_{j}}\left(\underline{\hat{x}}_{\text {out }, k}^{\text {det }}\right) \operatorname{Pr}\left(\underline{x}_{k}=\alpha_{j} \mid \Lambda_{\text {in }}^{\text {det }}\right)\right)}\right)
\end{array}
$$

with $\mathcal{A}_{i, \epsilon}=\left\{a \in \mathcal{A} \mid \boldsymbol{c}=\psi^{-1}(a), c_{i}=\epsilon\right\}$.

Let us mention that an empirical study proved that the expression of $\sigma_{\hat{x}}$ given by (6) keeps valid throughout the iterative process. The aforementioned a priori information is computed as follows:

$$
\operatorname{Pr}\left(\underline{x}_{k}=\alpha_{j} \mid \Lambda_{i n}^{\text {det }}\right)=\prod_{\substack{0 \leq i \leq m-1 \\ c^{(j)}=\psi^{-1}\left(\alpha_{j}\right)}} \operatorname{Pr}\left(c_{k m+i}=c_{i}^{(j)} \mid \Lambda_{i n}^{\text {det }}\right)
$$

and $\operatorname{Pr}\left(c_{k m+i}=c_{i}^{(j)} \mid \Lambda_{i n}^{\text {det }}\right)=\frac{\exp \left(u_{i, j} v_{k, i}\right)}{\exp \left(v_{k, i}\right)+\exp \left(-v_{k, i}\right)}$ with $u_{i, j}=2 c_{i}^{(j)}-1$ and $v_{k, i}=\frac{\Lambda_{i n}^{\text {det }}(k m+i)}{2}$.

By using a LogSumExp approximation [14] we can avoid saturation precision problems of the floating point, especially for high SNR and after some iterations.

The log likelihood ratio approximation reads

$$
\begin{aligned}
\Lambda_{\text {out }}^{\text {det }}(k m+i) & \approx \max _{\alpha_{j} \in \mathcal{A}_{i, 1}}\left(-\frac{\left(\underline{\hat{x}}_{\text {out }, k}^{\text {det }}-\alpha_{j}\right)^{2}}{2 \sigma_{\underline{\hat{x}}}^{2}}+u_{i, j} v_{k, i}\right) \\
& \left.-\max _{\alpha_{j} \in \mathcal{A}_{i, 0}}\left(-\frac{\left(\underline{\hat{x}}_{\text {out }, k}^{\text {det }}-\alpha_{j}\right)^{2}}{2 \sigma_{\underline{\hat{x}}}^{2}}\right)+u_{i, j} v_{k, i}\right)
\end{aligned}
$$

which is equivalent to

$$
\begin{aligned}
\Lambda_{\text {out }}^{\text {det }}(k m+i) \approx \max _{\alpha_{j} \in \mathcal{A}_{i, 1}}\left(\frac{\underline{\hat{x}}_{\text {out }, k}^{\text {det }} \alpha_{j}-\frac{1}{2} \alpha_{j}^{2}}{\sigma_{\underline{\hat{x}}}^{2}}+u_{i, j} v_{k, i}\right) \\
\\
-\max _{\alpha_{j} \in \mathcal{A}_{i, 0}}\left(\frac{\underline{\hat{x}}_{\text {out }, k}^{\text {det }} \alpha_{j}-\frac{1}{2} \alpha_{j}^{2}}{\sigma_{\underline{\hat{x}}}^{2}}+u_{i, j} v_{k, i}\right)
\end{aligned}
$$

Let $\Lambda_{i n}^{\text {dec }}$ be the sequence obtained after deinterleaving of $\Lambda_{\text {out }}^{\text {det }}$. We consider that the FEC code is a convolutional code and assume that the soft-in soft-out optimal Bahl Cocke Jelinek Raviv (BCJR) decoder [15] is used at the receiver. The FEC decoder produces $\Lambda_{\text {out }}^{\text {dec }}$. It can be decomposed as the sum of $\Lambda_{i n}^{d e c}$ and $\Lambda_{e x t}^{d e c}$. $\Lambda_{e x t}^{d e c}$ is extrinsic information. Let $\Lambda_{i n}^{\text {det }}$ be the result of interleaving of $\Lambda_{e x t}^{d e c}$. It is used as input of the binary-to-symbol converter (BSC) to provide a priori information to the detector in the following iteration.

Hereinafter, we propose two strategies to feed the detector with a priori information.

Strategy A: The first approach computes a soft decision based on the decoder output probabilities. The detector a priori information is denoted by $\underline{\hat{x}}_{i n}^{\text {det,A }}$ and is computed by

$$
\underline{\hat{x}}_{i n, k}^{\text {det }, A}=\sum_{\alpha_{j} \in \mathcal{A}} \alpha_{j} \operatorname{Pr}\left(\underline{x}_{k}=\alpha_{j} \mid \Lambda_{i n}^{\text {det }}\right)
$$

Strategy B: The second approach is based on hard decision. The detector a priori information denoted by $\underline{\hat{x}}_{i n}^{\text {det }, B}$ is equal to the symbol with the maximum probability at the decoder output:

$$
\underline{\hat{x}}_{i n, k}^{\text {det }, B}=\arg \max _{\alpha_{j} \in \mathcal{A}} \operatorname{Pr}\left(\underline{x}_{k}=\alpha_{j} \mid \Lambda_{i n}^{\text {det }}\right)
$$

\section{B. Detection criterion}

In order to take into account the a priori information delivered by the decoder in the proposed detector, we propose to resolve the optimization system with a regularization term as follows:

$\left(P_{R, 2}\right): \underset{\boldsymbol{B}_{1} \boldsymbol{r}=\mathbf{1}_{2 N}, \boldsymbol{r} \geq 0 .}{\arg \min }\left\|\underline{\boldsymbol{y}}-\underline{\boldsymbol{H}} \boldsymbol{B}_{\alpha} \boldsymbol{r}\right\|_{2}+\gamma\left\|\boldsymbol{B}_{\alpha} \boldsymbol{r}-\underline{\hat{\boldsymbol{x}}}_{i n}^{\text {det }}\right\|_{2}$ 


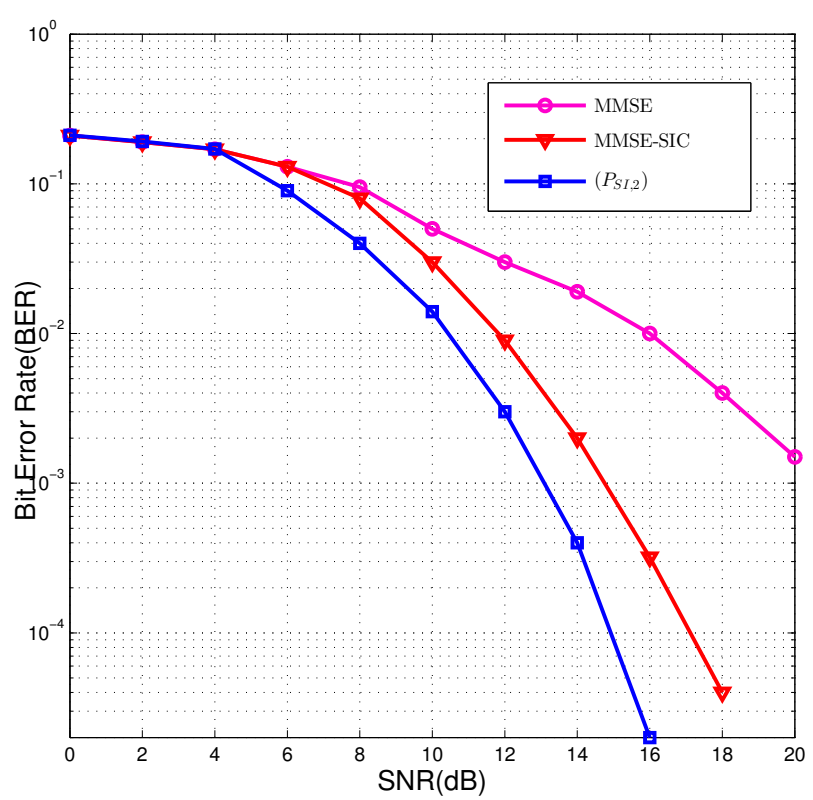

Fig. 5: BER performance comparison in $64 \times 64$ MIMO channel and 4-QAM.

where $\gamma$ is a positive weight less than 1 . It enables to take into account the unaccuracy of the estimated vector. The second term of regularization can be seen as a penalty, imposed to ensure that the detector output remains in the neighborhood of the decoder output.

\section{Choice of the regularization parameter}

The performance of our proposed detector depends highly on the choice of the regularization parameter. However, its optimization is an open issue. It depends on many parameters among which the SNR value and the probability that $\underline{\hat{x}}_{i n}^{\text {det }}$ is the searched vector (computed from the LLR provided by the decoder). In the future we intend to investigate the choice of $\gamma$. For the time being, in this article we consider a fixed value, independent of the SNR and of the current iteration.

\section{Simulation RESUlts}

\section{A. Non-Coded case}

In Fig. 5, we consider a $64 \times 64$ MIMO system with 4-QAM and we compare the $\left(P_{S I, 2}\right)$ detector to the MMSE-based detectors (which require $N \leq n$ ). The $\left(P_{S I, 2}\right)$ detector better exploits the receive diversity than the MMSE and MMSE-SIC detectors. At BER $10^{-2}$, the $\left(P_{S I, 2}\right)$ outperforms the MMSE by about $5.5 \mathrm{~dB}$ and the MMSE-SIC by $1.6 \mathrm{~dB}$. This gain increases and exceeds $7 \mathrm{~dB}$ and $2 \mathrm{~dB}$ respectively for a BER of $10^{-3}$.

Fig. 6 shows the performance for an underdetermined $16 \times 14$ MIMO system with 4-QAM. We observe that the proposed detector $\left(P_{S I, 2}\right)$ achieves a BER lower than $10^{-3}$ for the SNR values higher than $17 \mathrm{~dB}$. Beyond 8 $\mathrm{dB}$, the sphere decoder (SD) [16] outperforms $\left(P_{S I, 2}\right)$, e.g.,

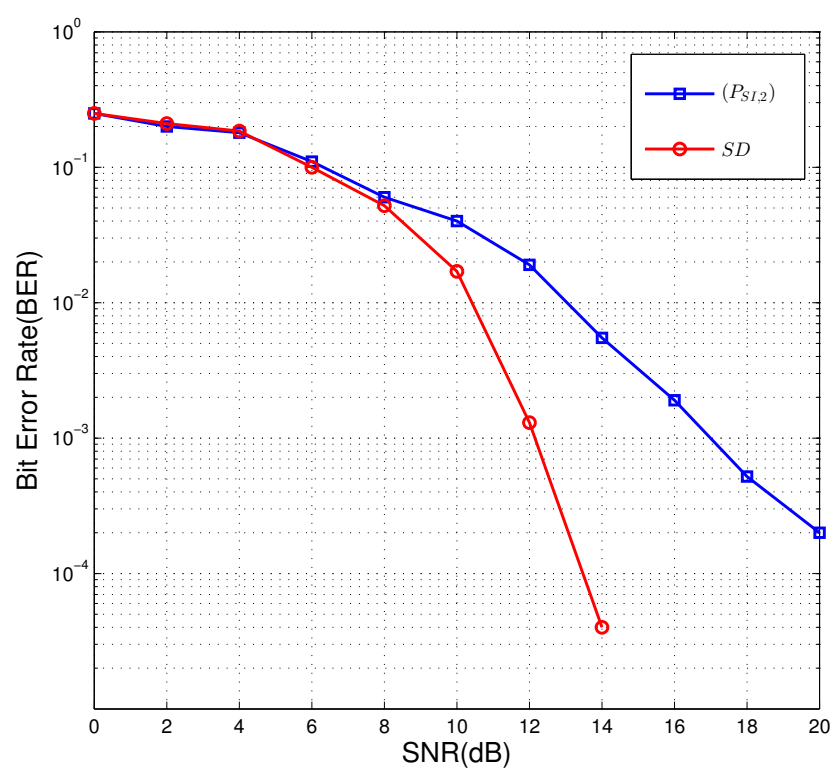

Fig. 6: BER performance comparison in $16 \times 14 \mathrm{MIMO}$ channel and 4-QAM.

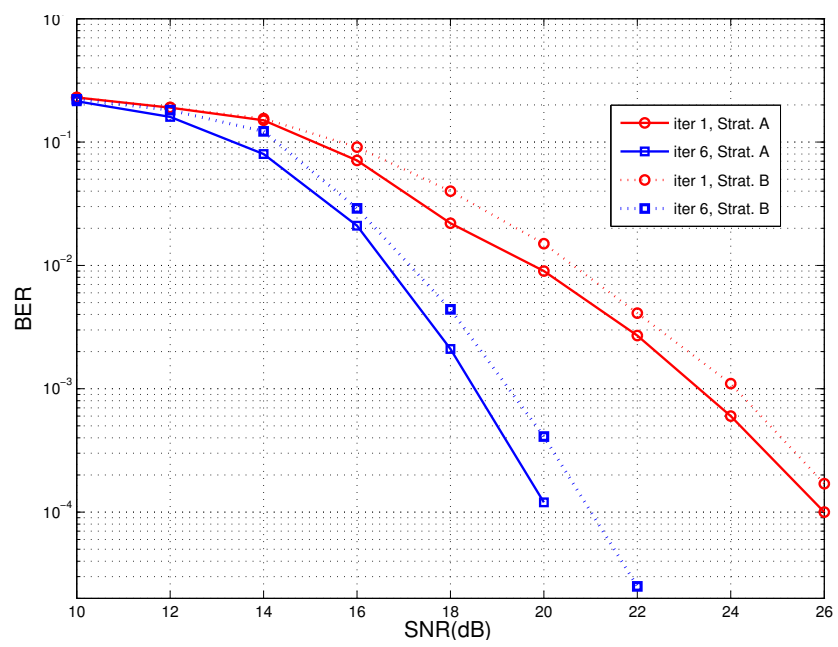

Fig. 7: BER performance comparison with $N=16$ and $n=$ 15 and 16-QAM (coded).

at BER $10^{-3}$, the gain is about $4.8 \mathrm{~dB}$. However as the MIMO system dimension increases, the SD computation cost will rapidly become too high to be implemented in practice, making the SD inadequate for large-scale MIMO applications.

\section{B. Coded case}

In this section, we compare the proposed two strategies for the turbo detection scheme. The convolutional code (CC) polynomials in octal are $(13,15)$ with a code rate equal to 0.5 . The regularization parameter in the detection criterion is empirically fixed to $\gamma=\frac{1}{3}$.

In Fig. 7, we consider $N=16, n=15, L=512$ 
(frame length) and $M=16$. We observe that the strategy Abased scheme outperforms the strategy B-based one. It can be seen that the gain increases with the SNR value. For instance, it achieves $1 \mathrm{~dB}$ at $\mathrm{BER}=10^{-4}$ at the sixth iteration. The efficiency of the exploitation of the extrinsic information between the detector and the decoder can be shown by looking at the improvement from the first iteration to the sixth one. For example, for strategy $\mathrm{A}$, it equals $6 \mathrm{~dB}$ at $\mathrm{BER}=10^{-4}$.

In [9], we proposed a detection scheme based on a sparse decomposition and we defined a turbo detection scheme, which compared positively with respect to a turbo-MMSE equalizer. Contrary to the current study, the soft input delivered to the FEC decoder in [9] was not based on the probability density function of the detector output which makes the turbo detector of [9] less efficient than the one proposed in this paper. We can thus predict that the latter should outperform the turbo-MMSE equalizer [17].

\section{CONCLUSION}

In this paper we have addressed the problem of detection in large MIMO systems with finite $M$-QAM constellation. We have proposed a real-valued formulation of the system model and then exploiting the vector simplicity we have defined the detection problem based on quadratic optimization with constraints to ensure the simplicity of the detected vector. We have given the conditions the underdetermined MIMO system must satisfy so that it can be detected successfully. Exploiting the probability density function of the detector output, we have computed a reliable soft input for a FEC decoder. We have also designed an iterative receiver based on a regularization method in the detection to further improve the BER performance.

\section{REFERENCES}

[1] D. L. Donoho and J. Tanner, "Counting the faces of randomly-projected hypercubes and orthants, with applications," Discrete \& Computational Geometry, vol. 43, no. 3, pp. 522-541, 2010.

[2] Z. Hajji, A. Aissa-El-Bey, and K. Amis, "Simplicity-based recovery of QAM signals for underdetermined large-scale MIMO," submitted to IEEE Transactions on Communications.

[3] Y. Saito, Y. Kishiyama, A. Benjebbour, T. Nakamura, A. Li, and K. Higuchi, "Non-orthogonal multiple access (noma) for cellular future radio access," in 2013 IEEE 77th Vehicular Technology Conference (VTC Spring), June 2013, pp. 1-5.

[4] B. Hassibi and H. Vikalo, "On the sphere-decoding algorithm I. expected complexity," IEEE Transactions Signal Processing, vol. 53, no. 8, pp. 2806-2818, Aug 2005.

[5] N. Kim, Y. Lee, and H. Park, "Performance analysis of MIMO system with linear MMSE receiver," IEEE Transactions on Wireless Communications, vol. 7, no. 11, pp. 4474-4478, November 2008.

[6] A. Aissa-El-Bey, D. Pastor, S. Sbai, and Y. Fadlallah, "Sparsity-based recovery of finite alphabet solutions to underdetermined linear systems," IEEE Transactions on Information Theory, vol. 61, no. 4, pp. 20082018, April 2015.

[7] Y. Fadlallah, A. Aissa-El-Bey, K. Amis, D. Pastor, and R. Pyndiah, "New iterative detector of MIMO transmission using sparse decomposition," IEEE Transactions on Vehicular Technology, vol. 64, no. 8, pp. 34583464, August 2015.

[8] — - "New decoding strategy for underdetermined MIMO transmission using sparse decomposition," in 2013 Proceedings of the 21st European Signal Processing Conference (EUSIPCO), September 2013, pp. 1-5.

[9] Z. Hajji, K. Amis, and A. Aissa-El-Bey, "Turbo detection based on sparse decomposition for massive mimo transmission," in 2016 9th International Symposium on Turbo Codes and Iterative Information Processing (ISTC), Sept 2016, pp. 290-294.
[10] D. L. Donoho and J. Tanner, "Counting faces of randomly-projected polytopes when the projection radically lowers dimension," Journal of the American Mathematical Society, vol. 22, no. 1, pp. 1-53, January 2009.

[11] H. Karloff, The Simplex Algorithm. Boston, MA: Birkhäuser Boston, 1991, pp. 23-47. [Online]. Available: http://dx.doi.org/10.1007/ 978-0-8176-4844-2_2

[12] Path-Following Interior-Point Methods, pp. 57-99. [Online]. Available: http://epubs.siam.org/doi/abs/10.1137/1.9781611970791.ch3

[13] B. Hassibi, "An efficient square-root algorithm for BLAST," in Acoustics, Speech, and Signal Processing, 2000. ICASSP '00. Proceedings. 2000 IEEE International Conference on, vol. 2, 2000, pp. II737-II740 vol.2.

[14] G. Calafiore and L. El Ghaoui, Optimization Models, ser. Control systems and optimization series. Cambridge University Press, October 2014.

[15] L. Bahl, J. Cocke, F. Jelinek, and J. Raviv, "Optimal decoding of linear codes for minimizing symbol error rate (corresp.)," IEEE Transactions on Information Theory, 1974.

[16] T. Cui and C. Tellambura, "An efficient generalized sphere decoder for rank-deficient mimo systems," IEEE Communications Letters, vol. 9, no. 5, pp. 423-425, 2005.

[17] C. Laot, R. L. Bidan, and D. Leroux, "Low-complexity MMSE turbo equalization: a possible solution for edge," IEEE Transactions on Wireless Communications, vol. 4, no. 3, pp. 965-974, 2005. 